\section{Bioenergie und nachhaltige Landnutzung}

von Daniela Thrän, Ludwig Leible, Sonja Simon, Sandro Pütz, Stefan Kälber, Christian Geiss und Christoph Schillings ${ }^{1}$

Bioenergie ist die wichtigste erneuerbare Energie und soll künftig weiter ausgebaut werden. Dies kann nur nachhaltig erfolgen, wenn die mit der Bioenergie verbundenen Landnutzungsfragen umfassend berücksichtigt werden. Nachhaltigkeitsindikatoren stellen dafür einen Ausgangspunkt dar, müssen jedoch die Anforderungen vor Ort umfassend berücksichtigen: So sind die ökologischen Folgen der Rohstoffbereitstellung nicht nur durch die klimatisch-physikalischen Voraussetzungen, sondern auch durch die konkrete kleinräumige und zeitliche Ausgestaltung bestimmt. Auf der Seite der Energiebereitstellung ist die Integration in die lokale Energienachfragestruktur entscheidend. Für Forschung und Politik ergibt sich hieraus die Forderung nach räumlich aufgelösten Informationen, Analysen und Steuerungsinstrumenten - sowohl für die Landnutzung als auch für die Energieplanung.

\section{Einleitung}

Traditionell ist Biomasse der größte Lieferant für erneuerbare Energien und stellte in 2009 mit 161 TWh an Wärme, Strom und Kraftstoff für Transport etwa $70 \%$ aller Erneuerbaren Energien in Deutschland bereit (Nitsch, Wenzel 2009). ${ }^{2}$ Biomasse ist mit $94 \%$ die dominierende erneuerbare Energiequelle im Wärmesektor.

Die Nutzung Erneuerbarer Energien wird stark zunehmen. Ihr Wachstum in der Vergangenheit, der Trend für die Zukunft und die bereits abgesteckten politischen Ziele weisen darauf hin. Welchen Anteil die Biomasse zukünftig zu einer nachhaltigen Energieversorgung in Deutschland beitragen kann, loten Szenarien aus, wie sie seit zehn Jahren vom Deutschen Institut für Luftund Raumfahrt (DLR) entwickelt werden. Diese berücksichtigen die Ziele der Bundesregierung zum Ausbau Erneuerbarer Energien (EE) und zur Steigerung der Energieeffizienz, die vorsehen, die Treibhausgasemissionen bis 2050 auf rund $20 \%$ des Werts von 1990 zu senken und den Beitrag der $\mathrm{EE}$ an der gesamten Energieversorgung auf rund $50 \%$ zu steigern. Wie Abbildung 1 zeigt, bleibt Biomasse danach auf absehbare Zeit der bedeutendste erneuerbare Energieträger in Deutschland

Abb. 1: Entwicklung des Endenergiebeitrags der Erneuerbaren Energien im Leitszenario 2010 bis 2050

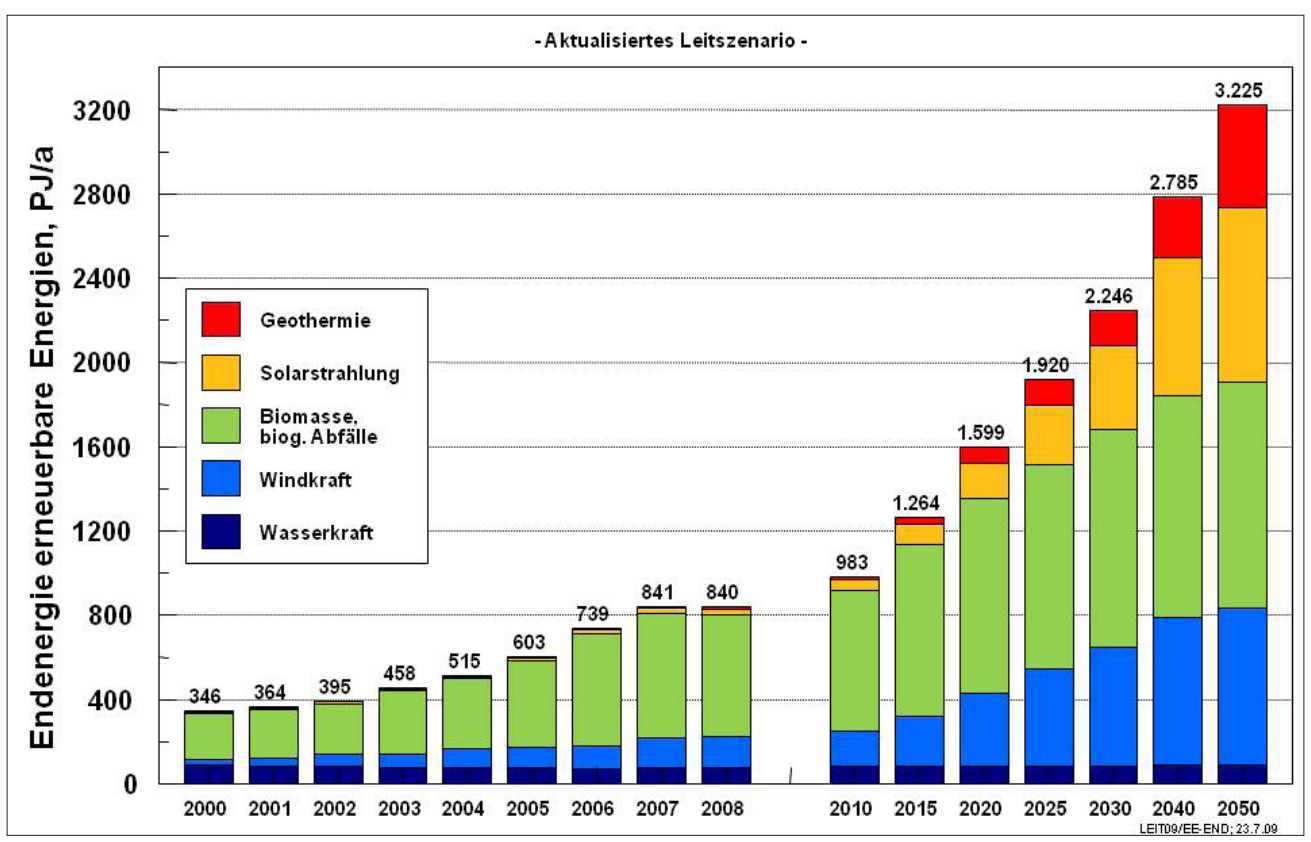

Quelle: Nitsch, Wenzel 2009, S. 8 
(Nitsch, Wenzel 2009). Insbesondere bis 2020 wird ein starker Zubau an Strom- und Wärmeproduktion aus Biomasse angenommen, der zu einem erheblichen Teil im Bereich der sehr effizienten Kraft-Wärme-Kopplung erzielt wird und zu über $90 \%$ auf den heimischen Rohstoffen basiert.

Das heimische Rohstoffpotenzial für die Bereitstellung von Bioenergie liegt in Deutschland im Bereich von 1.000 bis $1.400 \mathrm{PJ} / \mathrm{a}^{3}$, was etwa 10 bis $15 \%$ des nationalen Primärenergiebedarfs entspricht. Es umfasst etwa jeweils zu einem Drittel die Bereiche „Waldrestholz“, „Reststoffe aus Landwirtschaft, Industrie, Haushalten und dem kommunalen Bereich“ sowie „Energiepflanzen“ (Thrän, Kaltschmitt 2007). Letztere wurden im Jahr 2009 auf etwa zwei Mio. ha Ackerfläche angebaut und umfassten v. a. Raps und Biogassubstrate (FNR 2010). In verschiedener Hinsicht vorteilhaft ist künftig der Anbau mehrjähriger Pflanzen - weil hier günstigere Umwelteffekte erwartet werden und gleichzeitig die Deckung der zunehmenden Nachfrage nach holzartigen Biomassen unterstützt wird (Thrän et al. 2009). Gleichzeitig werden zudem vermehrte Rohstoffimporte mit entsprechenden Effekten auf Ökonomie, Ökologie und soziale Fragen in anderen Teilen der Welt erwartet.

\section{Anforderungen an eine nachhaltige Biomassebereitstellung und -nutzung}

Anforderungen an eine nachhaltige Biomassebereitstellung und -nutzung müssen - dem Leitbild „Nachhaltigkeit" folgend - die ökologische, ökonomische und soziale Dimension gleichrangig im Zielsystem zur Analyse und Bewertung abbilden. Teilweise wird zusätzlich auch die Berücksichtigung der institutionell-politischen Dimension gefordert, um das individuelle und kollektive Verhalten in Richtung Nachhaltigkeit $\mathrm{zu}$ verbessern ${ }^{4}$. Allgemeine Anforderungen, wie z. B. „nachhaltig“ oder ,umweltverträglich“, sind schnell formuliert. $\mathrm{Zu}$ deren Umsetzung ist jedoch eine aufwändige Differenzierung und Präzisierung von Bewertungsgrößen nötig, um den Chancen und Herausforderungen (TAB 2010) der Bioenergie gerecht zu werden.

Bei den aktuell gültigen Regelungen auf EUEbene bzw. auf nationaler Ebene beschränken sich die Anforderungen an die Nachhaltigkeit derzeit auf die ökologische Dimension und hierbei insbesondere auf das Merkmal „Treibhausgase“ bzw. auf die „Treibhausgas-Minderungspotenziale“ (vgl. Butterbach-Bahl et al. 2010). In diesem Zusammenhang wird auch auf den Schutz der biologischen Vielfalt hingewiesen. Unter diesem verengten Blickwinkel wurden 2009 mit der EURichtlinie 2009/28/EG (EU 2009) zur Förderung der Nutzung von Energie aus erneuerbaren Quellen erstmals Nachhaltigkeitskriterien für die Herstellung von Biomasse zur energetischen Verwendung vorgeschrieben und in 2010 mit der Einführung eines Zertifizierungssystems für nachhaltige Biokraftstoffe weitergehend präzisiert (EU 2010a; EU 2010b). Mit der Biomassestrom-Nachhaltigkeitsverordnung (BioSt-NachV 2009) und der Biokraftstoff-Nachhaltigkeitsverordnung (Biokraft-NachV 2009) sind diese Anforderungen in Deutschland bereits in nationales Recht umgesetzt.

Um sich dem komplexen Leitbild der Nachhaltigkeit schrittweise zu nähern, müssen dabei weitere Aspekte in die Bewertung der Bioenergieträger integriert werden. In Abbildung 2 wird der Versuch unternommen, an wesentlichen Aspekten darzustellen, welche Zusammenhänge bestehen und welche Anforderungen aber auch Auswirkungen prinzipiell berücksichtigt werden sollten, um eine abschließende Bewertung einer nachhaltigen Biomassebereitstellung und -nutzung durchführen zu können.

Wie in Abbildung 2 dargestellt, wird die Biomasse - neben der Nutzung als Energieträger - insbesondere stofflich als Lieferant von Nahrungs-/Futtermitteln und von Rohstoffen genutzt. Hierbei bestehen vielseitige Wechselwirkungen auf nationaler, EU- und globaler Ebene. Weiterhin sind die Auswirkungen der Bioenergienutzung konzeptionell und örtlich sehr unterschiedlich und nur in konkrete Anforderungen integrierbar, wenn sie durch entsprechende Bewertungsgrößen und Indikatoren abgebildet werden können. Landnutzungsfragen stellen dabei - infolge der vielfältigen ökologischen, ökonomischen, sozialen und institutionell-politischen Wechselwirkungen - einen zentralen Aspekt der nachhaltigen Biomassenutzung dar.

Räumlich hoch aufgelösten Untersuchungen zur Biomassebereitstellung (Biomassepotenziale) und zur Nutzung der Biomasse zur Wärmebereit- 
Abb. 2: Anforderungen und Auswirkungen einer nachhaltigen Biomassebereitstellung und -nutzung

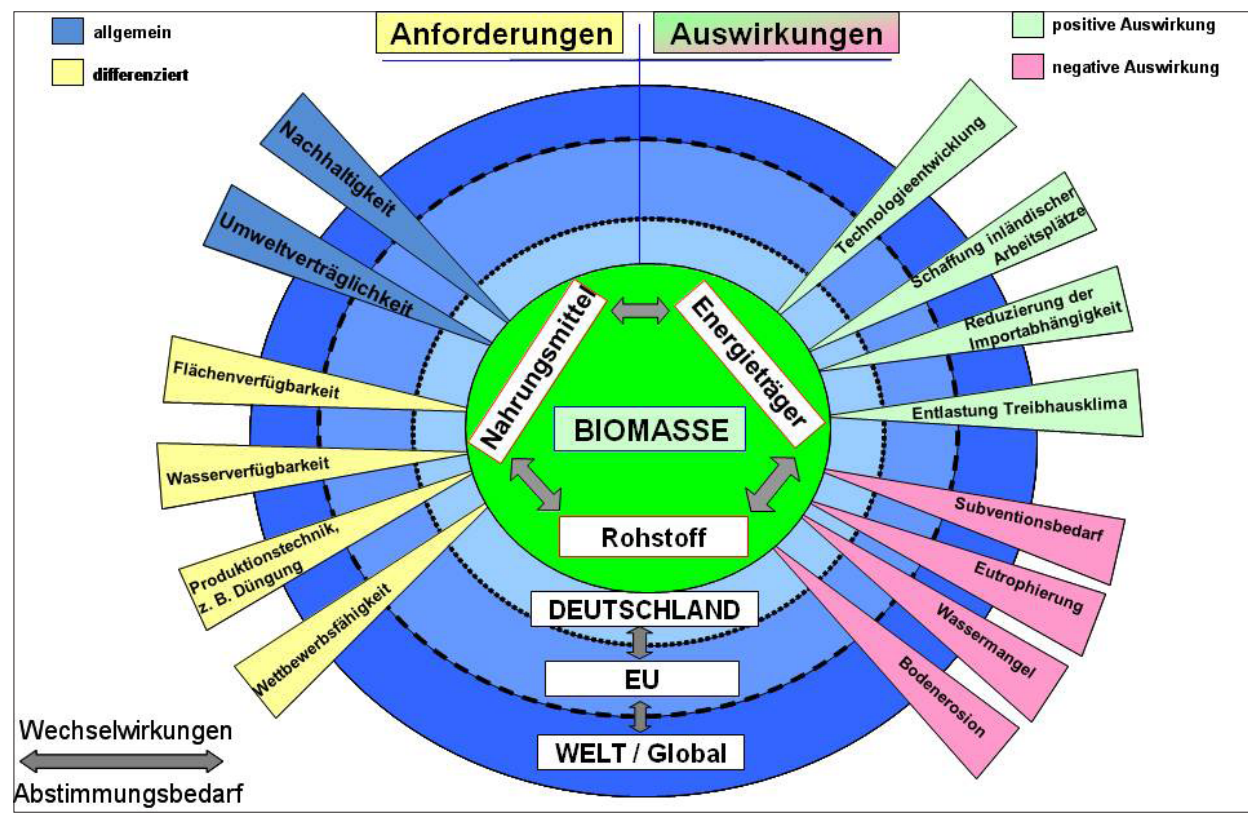

Quelle: Eigene Darstellung

stellung, insbesondere aber den mit der Biomassebereitstellung verbundenen ökologischen Folgen wird in den nachfolgenden Beispielen besonderes Augenmerk gewidmet. Anhand des Kriteriums „ $\mathrm{CO}_{2}$-Minderungskosten“ wird auf die Aspekte „Entlastung des Treibhausklimas“ und „Wettbewerbsfähigkeit" ausgewählter Bioenergie-Nutzungen bei der Verfolgung von TreibhausgasMinderungsstrategien eingegangen.

\section{Raumbezogene Effekte der Bioenergiebereitstellung}

\subsection{Regional verfügbare Biomassepotenziale}

Am KIT werden regional verfügbare Biomassepotenziale auf Basis der regionalspezifischen restriktiven Faktoren (z. B. Topografie, Umweltauflagen, Bodenfruchtbarkeit, Landnutzungsform, Besitzstruktur, u. a.) ermittelt; sie liefern Informationen über die räumliche Verteilung unterschiedlicher Biomassefraktionen. Beispielhaft ist dies in Abbildung 3 für das Aufkommen an Waldrestholz, Reststroh und Heu (von überschüssigem Grünland) in Baden-Württemberg darstellt. Die regionale Differenzierung als „mobilisierbares“
Biomasseaufkommen erfolgt $\mathrm{u}$. a. mit einem geografischen Informationssystem (GIS), basierend auf der spezifische Flächennutzung in den einzelnen Gemeinden (Kappler et al. 2010).

Die Analysen ergaben für Baden-Württemberg (Bezugsjahr 2007) ein jährliches Aufkommen von rd. 3 Mio. $\mathrm{Mg} \mathrm{TM}^{5}$ (s. Abb. 3); hiermit könnten rd. $3 \%$ des derzeitigen Primärenergiebedarfs gedeckt werden. Insgesamt könnten biogene Rest- und Abfallstoffe rd. $9 \%$ des Primärenergiebedarfs decken (Leible et al. 2008). Betrachtet man das für eine energetische Nutzung potenziell mobilisierbare Aufkommen an Waldrestholz (1,2 Mio. Mg TM), Reststroh (1,1 Mio. Mg TM) und Heu von überschüssigem Grünland (0,6 Mio. $\mathrm{Mg}$ TM), so resultiert - bezogen auf die Bodenfläche des Landes Baden-Württemberg (3,5 Mio. ha) eine durchschnittliche Aufkommensdichte von rd. $0,8 \mathrm{Mg} \mathrm{TM} / \mathrm{ha}$. Dabei bieten hinsichtlich des Strohs die Landkreise der Region Franken und DonauIller die größten Aufkommenspotenziale, während die größten Aufkommensdichten an Waldrestholz in den Landkreisen im Bereich des Schwarzwalds liegen. Die höchsten Aufkommensdichten für Heu von überschüssigem Grünland sind in den Regionen Neckar-Alb und Mittlerer Neckar zu finden. 
Abb. 3: Topografie und Aufkommensdichte an Waldrestholz, Reststroh und Heu nach Landkreisen und Regionalverbänden Baden-Württembergs

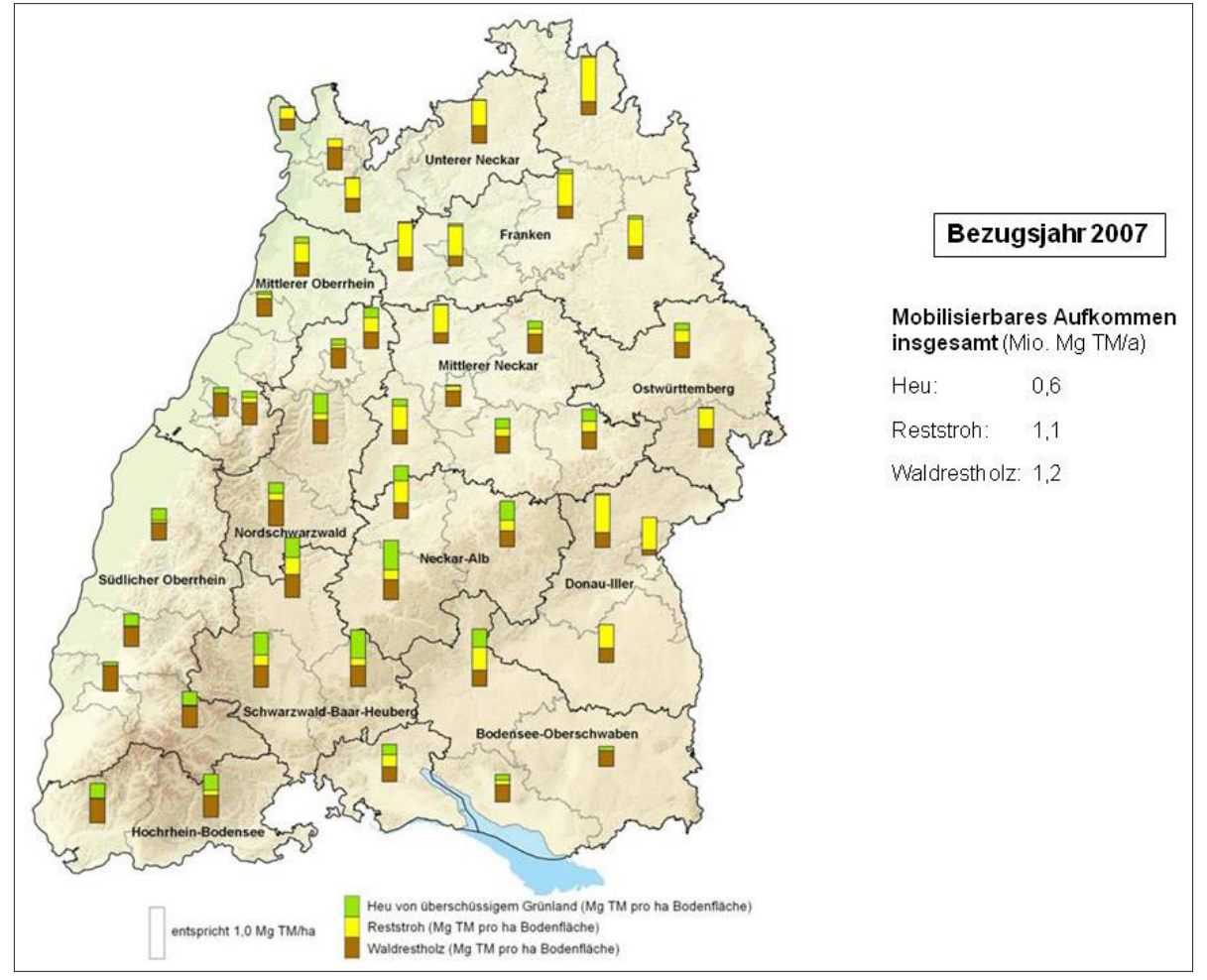

Quelle: Eigene Darstellung

\subsection{Abschätzung der ökologischen Folgen}

Die Berücksichtigung raumbezogener Aspekte erfordert mit Blick auf eine nachhaltige ökologisch orientierte Steuerung, dass die Wechselwirkungen von Anbausystemen mit ihrer belebten und unbelebten Umwelt verstanden und in langfristig nachhaltige Managementmethoden übersetzt werden können. Am UFZ stehen die räumliche und zeitliche Landnutzungsänderung als Dreh- und Angelpunkt auf der Skala des Schlages bis hin zur Region im Fokus, inkl. möglicher Landnutzungskonkurrenzen durch eine Zunahme des Biomasseanbaus. Eine lokale Landnutzungsänderung basiert auf der Landnutzungsentscheidung des Entscheidungsträgers, z. B. des Eigentümers, die von zahlreichen Faktoren abhängt, z. B. den Marktund gesetzlichen Rahmenbedingungen. Die Summe der lokalen Einzelentscheidungen ergeben eine räumlich spezifische regionale Landschaftsstruktur, z. B. die Flächenanteile der verschiedenen Landnutzungstypen, sowie eine spezifische Landschaftskonfiguration, z. B. die Größen- und räumliche Verteilung der Landnutzungstypen, in die eine zunehmende Nutzung der Bioenergie auf komplexe Weise einwirkt, indem sie die Landschaftsstruktur und -konfiguration verändert. Zahlreiche Umweltwirkungen der Bioenergienutzung sind räumlich verankert. ${ }^{6}$ Dazu gehören z. B. punktuelle Emissionsquellen, laterale Stoffflüsse sowie Interaktionen der belebten Umwelt mit der Landschaftsstruktur und -konfiguration oder mit anderen Arten. Durch die räumliche Verankerung und die daher möglichen Wirkungsgefüge kann es zu nichtlinearen räumlichen Effekten kommen (z. B. zu einer eingeschränkten Überlebensfähigkeit einer Population aufgrund von Randeffekten der Landschaftszerschneidung, zu einer fehlenden Mindestgröße oder zu einer zu starken Isolation von Habitaten). Solche Effekte, die sich erst auf Landschafts- und regionaler Ebene auswirken, können das Ergebnis einer multikriteriellen Umweltwirkungsanalyse mittels Nachhaltigkeitsindikatoren erheblich beeinflussen. Daher reicht es bei der Analyse der Umweltwirkungen oft nicht, allein von der Fläche aus hochzurechnen.

Zur Abschätzung solcher möglichen komplexen, nichtlinearen Umweltwirkungen durch 
zunehmende Nutzung der Bioenergie haben sich, neben dem Monitoring zur Datenaufnahme zusätzlich Stoffstrom- und statistische Modelle, sowie besonders räumlich explizite, mechanistische Simulationsmodelle in der Ökologie als hervorragendes neues Instrument bewährt, die empirisch schwer fassbare Langzeitfolgewirkungen menschlicher Landnutzung zu untersuchen. Letztere werden u. a. in der ökologischen Modellierung verwendet (Grimm et al. 2005; Grimm, Railsback 2005), sind aber genauso geeignet, um sozioökonomische Komponenten zu integrieren, wie sie bei der Nutzung der Bioenergie notwendig sind. Mechanistische (d. h. in aggregierter Form prozessbasierte), räumliche und oft individuen- bzw. agentenbasierte Simulationsmodelle erweitern die Optionen der Modellierung (v. a. der klassischen Modellen in der Ökologie), da diese die modellierten biologischen Organismen in ihrer raum-zeitlichen Dynamik realitätsnäher abbilden können (Grimm, Railsback 2005). ${ }^{7}$ Dies erfolgt zum Einen durch die Möglichkeit den zu modellierenden Organismen laterale Nachbarschaftsbeziehungen oder Interaktionen zuweisen zu können. Zum Anderen können empirisch messbare Variablen und Schlüsselprozesse integriert werden. Somit können sie u. a. auch das gesamte empirische Wissen für exemplarische Fragestellungen, z. B. der Überlebenswahrscheinlichkeit einer Art in einer bestimmten Landschaft, systematisch in ein konsistentes Modell integrieren. Eine Über- prüfung ist mit Feld- oder Fernerkundungsdaten möglich, falls diese mit den Variablen der Simulationsmodelle übereinstimmen. Sie können auch Hinweise auf zukünftigen Forschungsbedarf geben oder bei der Entwicklung von Managementstrategien zu Hilfe genommen werden.

Räumliche Effekte auf Schlag- oder Landschaftsebene wurden bereits erfolgreich mit räumlichen ökologischen Simulationsmodellen untersucht, wie z. B. der Effekt menschlicher Landnutzung auf Ökosysteme oder gefährdete Arten. Auf der Schlagebene wurde z. B. für Tropenwälder in Brasilien, einer sehr wichtigen Region für die globale Bioenergienutzung, gezeigt, dass kleinflächige Restwälder aufgrund von Landschaftszerschneidung, also einer „Fragmentierung", die auch durch zunehmende Biomassenutzung verursacht wird, ca. $50 \%$ ihrer Biomasse zusätzlich verlieren. Nach einer Waldrodung ändert sich an den neuen Waldrändern das Mikroklima, auf das empfindliche Baumarten mit höherer Sterblichkeit reagieren (Groeneveld et al. 2009). Pütz et al. (2010) zeigen, wie sich die Abnahme der Waldfragmentgröße durch den zunehmenden Grad der Landschaftszerschneidung negativ auf die mittlere gespeicherte Gesamtbiomasse auswirkt (s. Abb. 4). Huth et al. (2004) konnten mit Hilfe eines räumlichen Waldwachstumsmodells und einer multikriteriellen Analyse zeigen, dass schonender, selektiver Holzeinschlag im Vergleich zu konventionellem Holzeinschlag sowohl

\section{Abb. 4: Einfluss von Landschaftszerschneidung auf die langzeitliche Produktivität von} Tropenwaldfragmenten*

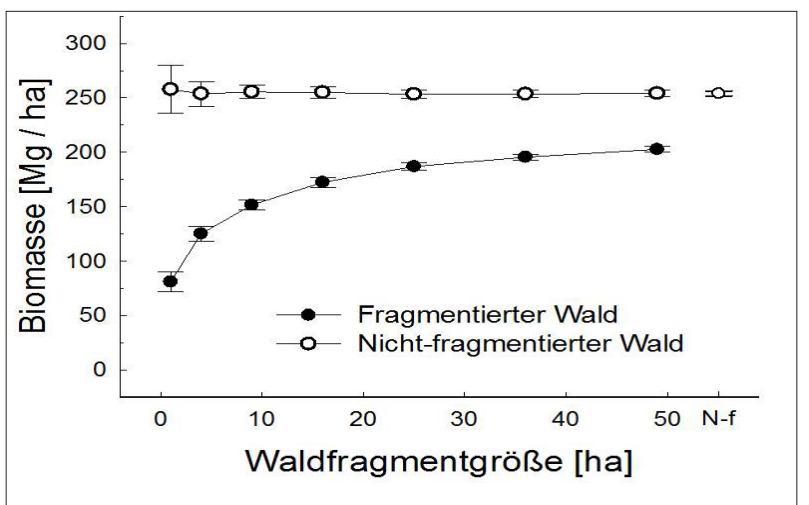

\footnotetext{
* = Mittlere in lebenden Bäumen gespeicherte Gesamtbiomasse (Trockenmasse) pro Hektar, in Abhängigkeit von der Fragmentgröße als indirektes Maß für den Grad der Landschaftszerschneidung (Fragmentierung)
}

Quelle: Pütz et al. 2010 
für den langfristigen Ernteertrag als auch für die Waldstruktur von Tropenwäldern vorteilhaft ist.

Bei einer angenommenen Zunahme der Biomassenutzung mit ihren Auswirkungen auf die Landnutzungsstruktur und -konfiguration sind räumliche Umwelteffekte auf der Landschaftsskala zu erwarten, die wie im Beispiel gezeigt, deutlich von der idealen Mittelwertsannahme abweichen dürften und daher mit Hilfe von räumlichen Simulationsmodellen untersucht werden sollten.

In Hinblick auf eine nachhaltige Biomassenutzung sollen mit den oben beschriebenen Methoden insbesondere folgende Fragestellungen untersucht werden: Wie kann ein langfristig nachhaltiger Biomasseanbau aussehen, wobei zur Bewertung der Umweltfolgen mehrere Umweltwirkungen berücksichtigt werden (z. B. lokale und laterale Stoffströme sowie Auswirkungen auf die belebte Umwelt). Wie wirken sich die Landschaftsstruktur bzw. deren Änderung sowie die Landschaftskonfiguration konkret auf die Nachhaltigkeit des Biomasseanbaus einer spezifischen Region aus? Wie könnte eine optimale Landnutzung unter Minimierung der negativen Folgen für Klima und Biodiversität sowie höchster Rohstoffernte aussehen?

\subsection{Nachhaltige Energiebedarfsdeckung durch Standortoptimierung}

Neben Bereitstellung und Auswirkung auf die Landnutzung stellt die Effizienz des Einsatzes die dritte Dimension einer nachhaltigen Bioenergienutzung dar. Für einen effizienten Einsatz der Biomasse sind geringe Energieverluste durch kurzen Transport und geringe Transformation der Biomasse von großer Bedeutung. Insbesondere bei der effizienten Kraft-Wärme-Kopplung ist die Lokalisation der Wärmenachfrage entscheidend für die Planung der Energiebereitstellung. Eine räumliche Auflösung dieser spezifischen ortsgebundenen Nachfrage kann als Entscheidungshilfe zur Technologie- und Standortauswahl für die vielseitig einsetzbare Biomasse dienen. Dies trifft insbesondere bei der Wärmebereitstellung zu.

Die am DLR entwickelten Nachfrageanalysen fokussieren auf die Ermittlung regionaler, räumlich hoch aufgelöster Nutzungspotenziale im Wärmesektor mit Hilfe von Fernerkundungs- daten. So kann bei entsprechender Nachfrage in einer Region die effiziente Wärmebereitstellung (z. B. in Nahwärmenetzen) einer Bereitstellung von Energiepflanzen für die Kraftstoffproduktion vorgezogen werden. Die Möglichkeit der Nutzung eines Wärmenetzes wird aufgrund des hohen Investitionsbedarfs primär von der Höhe des lokalen Wärmeabsatzes bedingt. Letzterer stellt wiederum einen idealtypischen Kennwert dar, der sich aus dem Gebäudetyp, der Nutzung des Gebäudes und dem Baualter ergibt (Fischedick et al. 2007; Kleeman et al. 2004; Sester et al. 2004). Für eine Bewertung des Nachfragepotenzials ist zusätzlich der wirtschaftliche Aufwand für die Erschließung des vorhandenen Wärmebedarfs zu berücksichtigen, also die infrastrukturellen Kosten der Nahwärmebereitstellung: Kosten für das Nahwärmenetz, Anschlussleitungen zu den einzelnen Gebäuden sowie Hausübergabestationen. Diese detaillierte Art der GIS-Datenanalyse stellt hohe Anforderungen an Datenqualität und räumliche Auflösung und ist v. a. für lokale Betrachtungen geeignet.

Auf dieser Grundlage lässt sich aus der Division des jährlichen Wärmebedarfs durch die benötigten, infrastrukturellen Investitionskosten ein Kennwert für die Standortgüte von Nahwärme ermitteln, der die durch das Versorgungssystem jährlich erschließbare Wärmemenge (kWh/ Jahr) pro investierter Geldeinheit $(€)$ repräsentiert. Die Potenzialwerte werden auf Baublockebene ausgewiesen (s. Abb. 5). Je höher dieser Wert ist, desto besser ist der jeweilige Baublock hinsichtlich seiner baulichen Physiognomie für eine Nahwärmeversorgung geeignet.

Für die Abschätzung der Nutzungspotenziale im Hinblick auf den Einsatz eines Energieträgers wie der Biomasse, wurde diese Methodik in aggregierter Form auf regionaler und nationaler Ebene eingesetzt (Fischedick et al. 2007).

Regional bestehen in Deutschland erhebliche Nutzungspotenziale für Biomasse, mit einem Schwerpunkt im Süden. In der Mitte Deutschlands finden sich hingegen eher weniger Regionen, die für die effiziente Nutzung der Biomasse in Kraftwärmekopplung und Nahwärmenetzen geeignet sind. Aufgrund der demografischen Entwicklung mit Bevölkerungsrückgang in vielen Regionen im Norden und Osten Deutschlands und stabiler bzw. wachsender Bevölkerung in einigen Regi- 
Abb. 5: Potenzialkarte für Wärmenetze in München

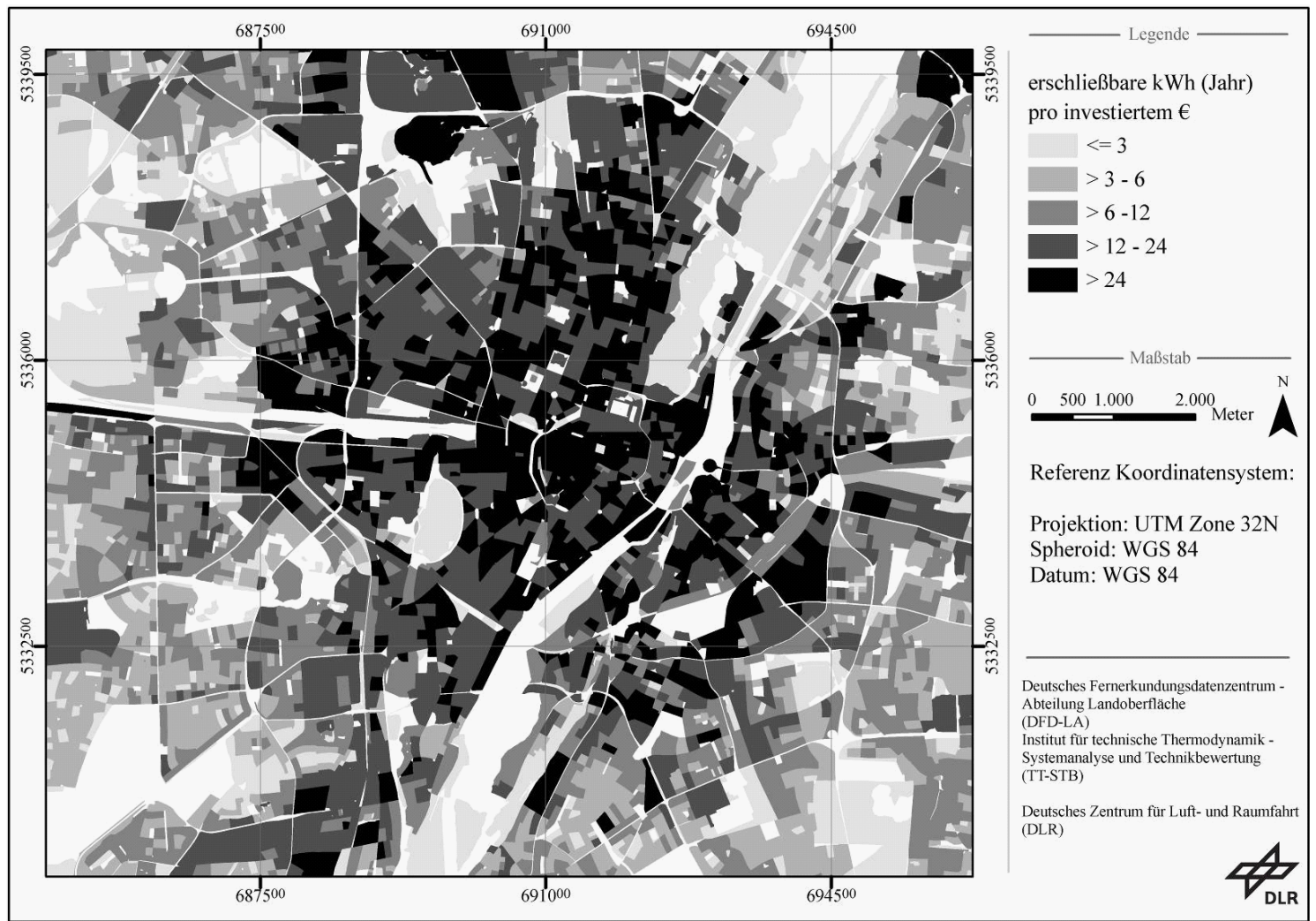

Quelle: DLR, eigene Darstellung in Anlehnung an Geiss et al. 2010

onen im Süden werden sich diese Unterschiede verstärken (Schillings, Simon 2011). Die Ergebnisse zeigen, dass eine dezentrale Energieplanung eine Grundvoraussetzung für eine nachhaltige Nutzung der Biomasse ist. Räumlich aufgelöste Nachfrageanalysen können einen erheblichen Beitrag zur effizienten und damit nachhaltigen Nutzung der Biomasse auf lokaler Ebene leisten.

\section{Treibhausgasminderung als raumüber- greifender Nachhaltigkeitsaspekt}

Neben der notwendigen stärkeren räumlichen Differenzierung besteht aber auch in raumübergreifenden Fragen noch Forschungs- und Entwicklungsbedarf. Wie in den Vorbemerkungen zum Leitbild „Nachhaltigkeit“ bereits erläutert, lässt sich eine Strategie zum Einsatz von Biomasse - im Vergleich zu anderen Erneuerbaren Energien oder Effizienzmaßnahmen - zur Entlastung des Treibhausklimas bzw. zur Klimastabilisierung am besten daran bemessen, welche Treibhausgas-Min- derungskosten damit verbunden sind. Die Kosten der Treibhausgas-Minderung ergeben sich aus den Mehrkosten und der erzielten Treibhausgas-Minderung im Vergleich zur fossilen Referenz.

Der Wissenschaftliche Beirat Agrarpolitik hat hierzu in seinem Gutachten „Nutzung von Biomasse zur Energiegewinnung - Empfehlungen an die Politik" eine umfangreiche Analyse durchgeführt und mit anderen Studienergebnissen verglichen (WBA 2008; Zimmer et al. 2008) (s. Abb. 6). Er kommt in seiner volkswirtschaftlichen Betrachtungsweise hinsichtlich der Verwendung von Biomasse zur Entlastung des Treibhausklimas in den Bereichen Wärme-, Strom- und Kraftstoffbereitstellung zu der nachfolgend skizzierten Wertung.

Die Wärme- bzw. die gekoppelte Wärmeund Stromerzeugung (KWK) über biogene Festbrennstoffe schneidet i. d. R. am günstigsten $a b$. Bei ihr werden die höchsten energetischen Gesamtnutzungsgrade und die geringsten Treibhausgas-Minderungskosten erreicht (s. Leible et al. 2008). Die gekoppelte Wärme-/Stromerzeugung 
Abb. 6: Treibhausgas-Minderungskosten bei der Gewinnung von Wärme, Strom und Kraftstoff aus Biomasse

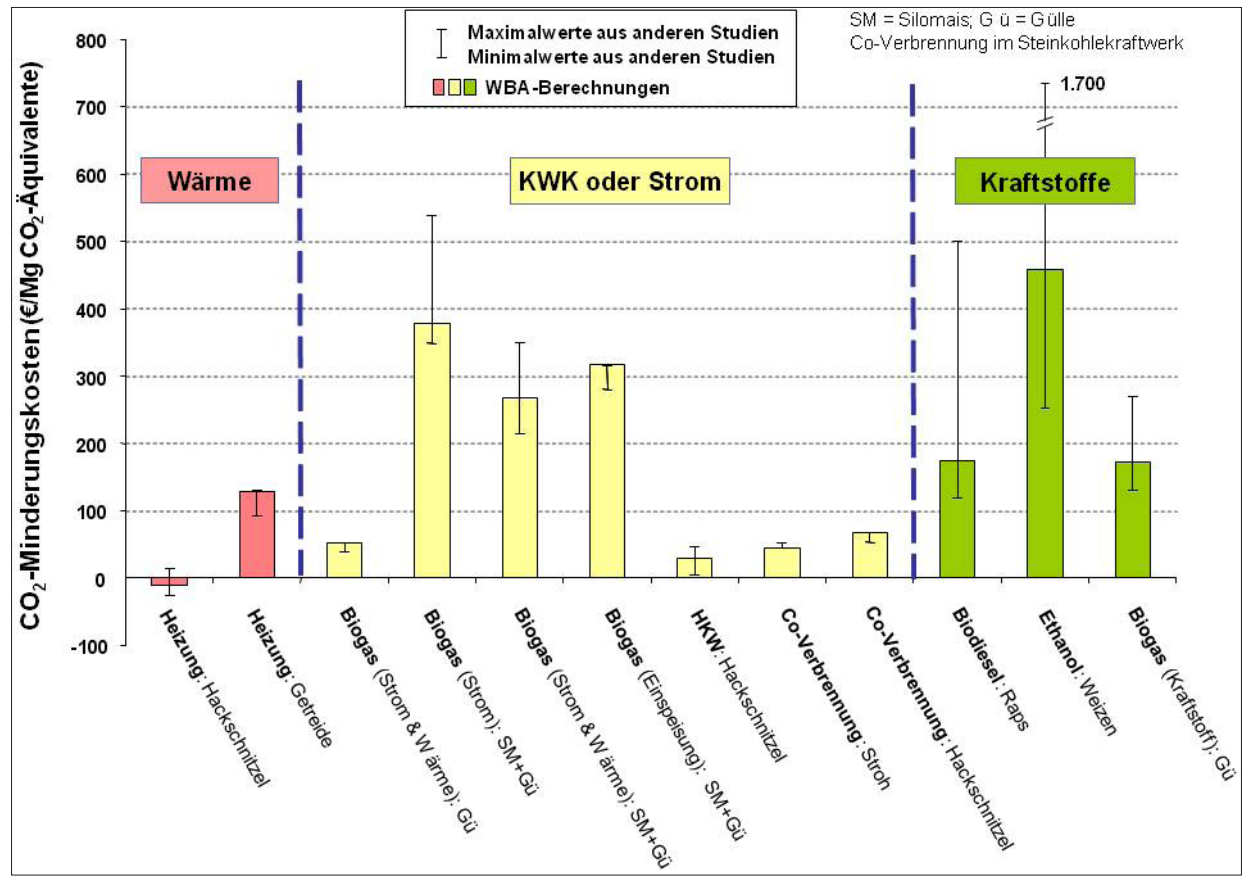

Quelle: WBA 2008; Zimmer et al. 2008; angepasst

über die Biogasgewinnung aus Gülle schneidet im Gegensatz zur Biogasgewinnung aus Silomais - ähnlich günstig ab, wie die Co-Verbrennung von Stroh in einem Steinkohlekraftwerk zur alleinigen Stromerzeugung. Dies ist ein Hinweis dahingehend, dass die energetische Nutzung von biogenen Rest- und Abfallstoffen in der Regel kostengünstiger ist und deutlich günstigere Treibhausgasbilanzen aufweist, verglichen mit angebauter Biomasse. Die Bereitstellung der Kraftstoffe Biodiesel aus Raps oder Ethanol aus Weizen schneidet bei diesem Vergleich besonders ungünstig ab.

Berücksichtigt man allerdings den erwarteten Wandel im Energiesystem, ergeben sich bei sinkendem Wärmebedarf und zunehmendem Anteil Erneuerbarer Energien an der Strombereitstellung - ggf. andere Rangfolgen in den eingesparten Treibhausgasen und damit auch veränderte Treibhausgas-Minderungskosten. Beim Vergleich zwischen Reststoffen und angebauten Energiepflanzen bestehen grundsätzliche Wettbewerbsvorteile für die reststoffbasierten Systeme (Stroh, Gülle, Hackschnitzel), weil die Treibhausgasemissionen, die mit dem Energiepflanzenanbau verbunden sind, die erreichbaren Treibhausgas-Minderungen reduzieren. Zudem besteht ge- genwärtig noch Unklarheit, in welchem Ausmaß es beim Anbau der Biomasse zur Emission des sehr klimawirksamen Lachgases $\left(\mathrm{N}_{2} \mathrm{O}\right)$ kommt oder die Kohlenstoffvorräte im Boden abgebaut werden; beide Effekte können entscheidende Auswirkungen auf die künftige Einordnung haben (Butterbach-Bahl et al. 2010).

\section{Nachrichten an Forschung und Politik}

Die energetische Biomassenutzung bedarf vielfältiger Optimierungen in Hinblick auf die nachhaltige Bereitstellung und Nutzung. Nachhaltigkeit als Zielsystem sollte keinesfalls nur auf die Dimension „Umwelt und Ökologie“ verengt werden; die ökonomische und soziale Dimension sind gleichrangig einzubeziehen.

Weiterhin sind viele Nachhaltigkeitseffekte räumlich differenziert zu betrachten und $\mathrm{zu}$ optimieren. Bei der Biomassebereitstellung ist eine hoch aufgelöste Analyse und Modellierung der Landnutzung notwendig, die Managementansätze für eine optimierte Biomassebereitstellung liefern können. Bei der Biomassenutzung ist insbesondere die räumliche Differenzierung der Nachfrage - vor dem Hintergrund optimierter Anlagenstand- 
orte - entscheidend. Während durch GIS heute die Möglichkeiten zur zeitnahen Bereitstellung und Verarbeitung hoch aufgelöster Daten gegeben sind und weitere Fortschritte erwartet werden können, bestehen bei der Modellierung der Wechselwirkungen und bei der regionalen Steuerung noch viele ungeklärte Fragen. Offen ist hierbei insbesondere, ob und wie Landnutzung durch Bioenergie nachhaltiger gestaltet werden kann.

An die Politik geht vor diesem Hintergrund die Empfehlung (s. TAB 2010), die punktuell begonnenen Nachhaltigkeitsanforderungen für Bioenergie systematisch zu erweitern und über das System Bioenergie hinaus die gesamte Landnutzung einzubeziehen. $\mathrm{Zu}$ fordern sind Steuerungssysteme, die sicherstellen, dass Biomasse für die Bereitstellung von Nahrungsmitteln genutzt wird; hierbei sollten für alle Nutzungsformen ähnliche Anforderungen an die Nachhaltigkeit gelten. Schließlich bringt auch die regional spezifizierte Steuerungsnotwendigkeit - sowohl für den Biomasseanbau als auch für die Biomassenutzung - neue Anforderungen an eine stärker dezentral ausgerichtete Energieplanung, für die es gegenwärtig kaum geeignete Instrumente gibt.

\section{Anmerkungen}

1) Sandro Pütz und Daniela Thrän forschen am Helmholtz-Zentrum für Umweltforschung (Leipzig), Ludwig Leible und Stefan Kälber am Institut für Technikfolgenabschätzung und Systemanalyse am KIT, Sonja Simon, Christian Geiss und Christoph Schillings am Deutschen Institut für Luft- und Raumfahrt (Stuttgart). Daniela Thrän arbeitet auch am Deutschen BiomasseForschungsZentrum (Leipzig).

2) Die Erneuerbaren Energien insgesamt erzeugten in $2009233 \mathrm{TWh}$.

3) $1 \mathrm{PJ}=10^{15}$ Joule; zur Einordnung: Eine Tonne trockene Biomasse (Mg TM) entspricht energetisch rd. 17 GJ; folglich entspricht 1 PJ rd. 60.000 t trockener Biomasse.

4) Vgl. Grunwald, Kopfmüller 2006 und Schultz et al. 2008.

5) $\mathrm{Mg} \mathrm{TM}$ : Megagramm $\left(=10^{6} \mathrm{~g}=1000 \mathrm{~kg}=1\right.$ Tonne); $\mathrm{TM}=$ Trockenmasse, Umrechnung in Energieeinheiten siehe Anmerkung 1.

6) Einen Überblick dazu geben Thrän, Gawor 2011.

7) Weitere Diskussion modellierspezifischer Fragen siehe Grimm, Railsback 2005.

\section{Literatur}

Biokraft-NachV, 2009: Verordnung über Anforderungen an eine nachhaltige Herstellung von Biokraftstoffen (Biokraftstoff-Nachhaltigkeitsverordnung - Biokraft-NachV), vom 30. September 2009. BGB1 I (65), S. 3182-3212

BioSt-NachV, 2009: Verordnung über Anforderungen an eine nachhaltige Herstellung von flüssiger Biomasse zur Stromerzeugung (Biomassestrom-Nachhaltigkeitsverordnung - BioSt-NachV), vom 23. Juli 2009. BGB1 I (46), S. 2174-2202

Butterbach-Bahl, K.; Leible, L.; Kälber, S. et al., 2010: Treibhausgasbilanz nachwachsender Rohstoffe - eine wissenschaftliche Kurzdarstellung. KIT Scientific Reports 7556, ISBN 978-3-86644-532-1, Karlsruher Institut für Technologie (KIT), KIT Scientific Publishing, Karlsruhe; http://www.itas.fzk.de/deu/lit/2010/buua10a. pdf (download 20.9.10)

EU - Europäische Union, 2010a: Mitteilung der Kommission zu freiwilligen Regelungen und Standardwerten im Rahmen des EU-Nachhaltigkeitskonzepts für Biokraftstoffe und flüssige Biobrennstoffe. Amtsblatt der Europäischen Union, C160/1-7, 19.06.2010

EU-Europäische Union, 2010b: Mitteilung der Kommission zur praktischen Umsetzung des EU-Nachhaltigkeitskonzepts für Biokraftstoffe und flüssige Biobrennstoffe sowie zu den Berechnungsregeln für Biokraftstoffe. Amtsblatt der Europäischen Union, C160/8-16, 19.06.2010

EU - Europäische Union, 2009: Richtlinie des Europäischen Parlaments und des Rates vom 23. April 2009 zur Förderung der Nutzung von Energie aus erneuerbaren Quellen und zur Änderung und anschließenden Aufhebung der Richtlinien 2001/77/EG und 2003/30/ EG. Amtsblatt der Europäischen Union, L140/16-62, 05.06.2009

Fischedick, M.; Schüwer, D.; Venjakob, J. et al., 2007: Anforderungen an Nah- und Fernwärmenetze sowie Strategien für Marktakteure in Hinblick auf die Erreichung der Klimaschutzziele der Bundesregierung bis zum Jahr 2020. Wuppertal

FNR - Fachagentur Nachwachsende Rohstoffe, 2010: http://www.bmelv.de/SharedDocs/Standardartikel/ Landwirtschaft/Bioenergie-NachwachsendeRohstoffe/ NachwachsendeRohstoffe/FlaechenNutzungskonkurrenz.html (download 19.10.10)

Geiss, C.; Nast, M.; Schillings, C. et al., 2010: Potenzialmodellierung von Wärmenetzen basierend auf höchst aufgelösten Fernerkundungsdaten. In: Taubenböck, H.; Dech, S. (Hg.): Fernerkundung im urbanen Raum - Die Erdbeobachtung auf dem Weg zur Planungspraxis. Darmstadt

Grimm, V.; Railsback, S.F., 2005: Individual-Based Modeling and Ecology. Princeton

Grimm, V.; Revilla, E.; Berger, U. et al., 2005: Patternoriented Modeling of Agent-based Complex Systems: 
Lessons from Ecology. In: Science 310/5750 (2005), S. 987-991; DOI: 10.1126/science.1116681

Groeneveld, J.; Alves, L.F.; Bernacci, L.C. et al., 2009: The Impact of Fragmentation and Density Regulation on Forest Succession in the Atlantic Rain Forest. In: Ecological Modelling 220/19 (2009), S. 2450-2459

Grunwald, A.; Kopfmüller, J., 2006: Nachhaltigkeit. Campus-Einführungen. Frankfurt a. M.

Huth, A.; Drechsler, M.; Köhler, P., 2004: Multicriteria Evaluation of Simulated Logging Scenarios in a Tropical Rain Forest. In: Journal of Environmental Management 71/4 (2004), S. 321-333

Kappler, G.; Koch, B.; Leible, L., 2010: Wald-Energieholzaufkommen in Baden-Württemberg - Bereitstellungskosten und Standortanalyse. In: Allgemeine Forst und Jagdzeitung 181/5-6 (2010), S. 117-122

Kleemann, M.; Krüger, B.; Heckler, R., 2004: Verbrauchskennzahlen für Wohn- und Nichtwohngebäude in Städten. In: Lutsch, W., Neuffer, H., Witterhold, F.-G. (Hg.): Strategien und Technologien einer pluralistischen Fern- und Nahwärmeversorgung in einem liberalisierten Energiemarkt unter besonderer Berücksichtigung der Kraft-Wärme-Kopplung und regenerativer Energien. Frankfurt a. M., AGFW-Hauptstudie- Erster Bearbeitungsschritt, Band 1, S. 25-131

Leible, L.; Kälber, S.; Kappler, G., 2008: Energiebereitstellung aus Stroh und Waldrestholz. In: BWK 5 (2008), S. 56-62; http://www.itas.fzk.de/deu/lit/2008/leua08a. pdf (download 19.10.10)

Nitsch, J.; Wenzel, B., 2009: Leitszenario 2009 - Langfristszenarien und Strategien für den Ausbau erneuerbarer Energien in Deutschland unter Berücksichtigung der europäischen und globalen Entwicklung. Berlin: Bundesministerium für Umwelt, Naturschutz und Reaktorsicherheit, Reihe Umweltpolitik; http://www.bmu.de/files/ pdfs/allgemein/application/pdf/leitszenario2009_bf.pdf (download 22.11.10)

Pütz, S.; Groeneveld, J.; Alves, L.F. et al., 2010/i. E.: Fragmentation Drives Tropical Forest Fragments to Early Successional States: A Modeling Study for Brazilian Atlantic Forests. In: Ecological Modelling/eingereicht

Sester, M.; Neidhart, H.; Schulz, W. et al., 2004: Verfahrensentwicklung zur Bestimmung einer digitalen Wärmebedarfskarte aus Laserscanning- und GIS-Daten. In: Lutsch, W.; Neuffer, H.; Witterhold, F.-G. (Hg.): Strategien und Technologien einer pluralistischen Fern- und Nahwärmeversorgung in einem liberalisierten Energiemarkt unter besonderer Berücksichtigung der KraftWärme-Kopplung und regenerativer Energien. AGFWHauptstudie - Erster Bearbeitungsschritt, Band 1. Frankfurt a. M., S. 235-301

Schillings, C.; Simon, S., 2011/i. E.: The Potential for District Heating Based on Renewable Energy: A Spatial Analysis. In: Kuckshinrichs, W.; Kronenberg, T. (Hg): Demogra- phy and Infrastructures: National and Regional Aspects of Demographic Change. Berlin, in print

Schultz, J.; Brand, F.; Kopfmüller, J.; Ott, K., 2008: Building a Theory of Sustainable Development: Two Salient Conceptions within the German Discourse. In: International Journal of Environment and Sustainable Development 7/4 (2008), S. 465-482

TAB - Büro für Technikfolgen-Abschätzung beim Deutschen Bundestag, 2010: Chancen und Herausforderungen neuer Energiepflanzen (Autoren: Meyer, R.; Rösch, Chr.; Sauter, A.). Endbericht, TAB-Arbeitsbericht Nr. 136, Berlin

Thrän, D.; Edel, M.; Seidenberger, T. et al., 2009: Identifizierung strategischer Hemmnisse und Entwicklung von Lösungsansätzen zur Reduzierung der Nutzungskonkurrenzen beim weiteren Ausbau der energetischen Biomassenutzung. 1. Zwischenbericht, Leipzig

Thrän, D.; Gawor, M., 2011/i. E.: Biomass Provision and Use - Sustainability Aspects, Encyclopaedia of Sustainability Science and Technology, in print

Thrän, D.; Kaltschmitt M., 2007: Competition - Supporting or Preventing an Increased Use of Bioenergy? In: Biotechnology Journal 2/12 (2007), S. 1514-1524

WBA - Wissenschaftlicher Beirat Agrarpolitik beim Bundesministerium für Ernährung, Landwirtschaft und Verbraucherschutz, 2008: Nutzung von Biomasse zur Energiegewinnung - Empfehlungen an die Politik (verabschiedet: Nov. 2007; Erscheinungsdatum: 24.01.2008); DOI: 10.1002/biot.200700162; http://www.bmelv.de/cae/servlet/ contentblob/382594/publicationFile/23017/GutachtenWBA.pdf (download 19.10.10)

Zimmer, Y.; Berenz, S.; Döhler, H. et al., 2008: Klimaund energiepolitische Analyse ausgewählter Bioenergie-Linien. Landbauforschung, Sonderheft 318, $120 \mathrm{~S}$.; http://www.bfafh.de/bibl/lbf-pdf/landbauforschung-sh/ lbf_sh318.pdf (download 19.10.10)

\section{Kontakt}

Dr. Daniela Thrän

DBFZ und UFZ

Torgauer Straße 116, 04347 Leipzig

E-Mail: daniela.thraen@dbfz.de

Dr. Ludwig Leible

Karlsruher Institut für Technologie (KIT)

Institut für Technikfolgenabschätzung und

Systemanalyse (ITAS)

Campus Nord

Hermann-von-Helmholtz-Platz 1

76344 Eggenstein-Leopoldshafen

Tel.: +49 (0) 7247 / 82 - 4869

E-Mail: ludwig.leible@kit.edu 